\title{
Evaluación de la gravedad en la neumonía del adulto adquirida en la comunidad
}

\author{
FERNANDO SALDÍAS P.* y JUANA PAVIÉ G.**
}

\author{
EVALUATING SEVERITY \\ OF COMMUNITY-ACQUIRED PNEUMONIA IN ADULTS
}

\begin{abstract}
Clinical evolution in patients affected by community acquired pneumonia varies from a mild and low risk infectious disease to an extremely severe, life threatening disease. Commonly, immunocompetent adults without co-morbidities or severe risk factors cared for at out patient clinic have low risk of complications and death (mortality below 1-2\%); it increases to 5-15\% in patients with co-morbidities and/or with specific risk factors that are admitted into the hospital and reaches 20-50\% in those patients admitted into ICUs. Evaluation of severity in patients with pneumonia allows the prediction of disease evolution, establishing the proper setting of care, the type- of microbiological tests needed, and to choose the best empiric antibiotic treatment. It is suggested that patients be in three risk categories: low risk (mortality under 1-2\%) susceptible to ambulatory treatment; high risk patients (mortality 20$30 \%)$ that need specialized wards; and intermediate risk patients, with co-morbidities and/or risk factors for complicated clinical evolution and death, but cannot be classified in a specific category. In the ambulatory setting, without availability of complete laboratory exams, it is recommended to evaluating the severity of pneumonia considering the following clinical variables: age over 65 years, presence of comorbidities, sensorial compromise, vital signs alteration, degree of radiological involvement: multilobar, bilateral findings, cavitations), pleural effusion and arterial oximetry $<90 \%$. However, clinical judgement and the physician's experience must predominate over predictive models, which are not infallible.
\end{abstract}

Key words: pneumonia, prognosis, severity of illness index.

\section{RESUMEN}

La evolución del paciente con neumonía adquirida en la comunidad puede variar entre un cuadro infeccioso banal de bajo riesgo de complicaciones hasta uno de extrema gravedad con riesgo vital. En general, el adulto inmunocompetente sin co-morbilidad ni criterios de gravedad manejado en el medio ambulatorio tiene bajo riesgo de complicaciones y muerte (letalidad menor de 1-2\%), elevándose a 5$15 \%$ en los pacientes con co-morbilidad y/o factores de riesgo específicos que son admitidos al hospital y a 20-50\% en aquellos admitidos a la Unidad de Cuidados Intensivos. La evaluación de la gravedad en el paciente con neumonía permite predecir la evolución de la enfermedad, orientar el lugar de manejo, la extensión del estudio microbiológico, y el tratamiento antimicrobiano empírico. Se sugiere clasificar a los enfermos en tres categorías de riesgo: pacientes con bajo riesgo (mortalidad inferior a 1-2\%) susceptibles de tratamiento ambulatorio; pacientes con

\footnotetext{
* Departamento de Enfermedades Respiratorias, Facultad de Medicina. Pontificia Universidad Católica de Chile, Santiago, Chile.

** Servicio de Medicina, Hospital San Martín de Quillota, Chile.
} 
alto riesgo (mortalidad entre 20-30\%) que deben ser manejados en unidades especializadas del hospital; y pacientes con riesgo intermedio, con co-morbilidad y/o factores de riesgo de evolución complicada y muerte, pero que no es posible clasificar en una categoría precisa. En el medio ambulatorio, donde no se dispone de exámenes complementarios, se recomienda evaluar la gravedad de los pacientes con neumonía considerando las siguientes variables clínicas: edad sobre 65 años, presencia de co-morbilidad, compromiso de conciencia, alteración de los signos vitales, compromiso radiográfico multilobar o bilateral, presencia de cavitación o efusión pleural, y $\mathrm{SaO}_{2}<90 \%$. Sin embargo, el juicio clínico y la experiencia del médico deben predominar sobre los modelos predictores, los cuales no son infalibles.

Palabras clave: neumonía, pronóstico, evaluación de gravedad del paciente.

\section{EVALUACIÓN DE LA GRAVEDAD EN LA NEUMONÍA ADQUIRIDA EN LA COMUNIDAD}

El cuadro clínico del paciente con neumonía adquirida en la comunidad (NAC) que solicita atención en el medio ambulatorio (consultorios y servicios de urgencia) puede variar entre un cuadro infeccioso leve de bajo riesgo de complicaciones hasta uno de extrema gravedad con riesgo vital ${ }^{1-3}$ [Ib]. El amplio espectro de gravedad en la presentación de los enfermos explica la extensa variación en las tasas de letalidad por neumonía comunicadas en la literatura nacional e internacional en distintos contextos clínicos ${ }^{4}$. Así, el paciente con neumonía que no presenta factores de riesgo manejado en el medio ambulatorio tiene una letalidad inferior al $1-2 \%$, elevándose a $5-15 \%$ en los pacientes con comorbilidad y/o factores de riesgo específicos que son admitidos al hospital, y asciende a 20$50 \%$ en aquellos admitidos a la Unidad de Cuidados Intensivos (UCI) ${ }^{4}$ [Ia]. Una vez establecido el diagnóstico de neumonía, la evaluación de la gravedad constituye la principal preocupación del médico, lo cual le permitirá decidir el lugar de manejo (ambulatorio o en el hospital: sala de cuidados generales, Unidad de Intermedio o UCI), la extensión del estudio microbiológico y de laboratorio, la cobertura del tratamiento antibiótico empírico, la vía de administración y duración del tratamiento, y el nivel de los cuidados médicos y de enfermería que requiere el caso particular. La identificación de los enfermos de bajo riesgo de complicaciones susceptibles de ser manejados en el medio ambulatorio permitiría reducir significativamente los costos de la atención de salud, minimizando los riesgos asociados, sin comprometer la evolución y pro-

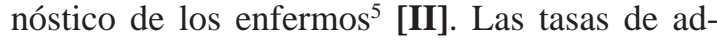
misión hospitalaria de pacientes adultos con NAC comunicadas en la literatura varían considerablemente, lo cual sugiere que los médicos no utilizan criterios uniformes para valorar el riesgo de morbilidad y mortalidad de los enfermos. Se ha comunicado que los médicos con frecuencia sobrestiman el riesgo de complicaciones y muerte de los pacientes con NAC y consecuentemente hospitalizan un número importante de pacientes de bajo riesgo ${ }^{6}[\mathbf{I I I}]$. La estratificación objetiva del riesgo de los pacientes debería ayudar a reducir esta variabilidad y mejorar la decisión de ingreso así como el manejo costo efectivo de la enfermedad.

\section{FACTORES PRONÓSTICOS EN LA NEUMONÍA ADQUIRIDA EN LA COMUNIDAD: VARIABLES CLIINICAS, RADIOGRÁFICAS Y DE LABORATORIO ASOCIADAS A EVOLUCIÓN CLÍNICA DESFAVORABLE Y MORTALIDAD ELEVADA}

Numerosos estudios han examinado los factores de riesgo en la admisión al hospital o a la UCI asociados a evolución clínica complicada y/o mal pronóstico vital, especialmente referido a la mortalidad en el hospital ${ }^{1-4}$ [Ia, Ib, II, III]. Los estudios univariados han descrito más de 40 índices clínicos y de laboratorio asociados con la mortalidad (Tabla 1). Sin embargo, una asociación independiente con el riesgo de muerte ha sido encontrada sólo para algunas variables clínicas mediante análisis multivariado.

Para facilitar el manejo de los factores pronósticos en neumonía, es conveniente agruparlos en distintas categorías ${ }^{1-4}$ : variables sociodemográficas (edad, lugar de procedencia, factores sociales), historia clínica (presencia de comorbilidad, estado mental alterado, fiebre, tos, disnea, escalofríos, dolor torácico), examen físico (hipotensión arterial, taquipnea, hipotermia, hipertermia, confusión mental), radiografía de tórax (infiltrados radiográficos multilobares o bilaterales, presencia de cavitación o derrame 
Tabla 1. Factores pronósticos asociados con evolución clínica complicada y riesgo de muerte en adultos inmunocompetentes con neumonía adquirida en la comunidad ${ }^{1-4,7,8}$

- Mayores de 65 años (población senescente)

- Comorbilidad: cardiopatía coronaria, insuficiencia cardíaca congestiva, enfermedad pulmonar crónica (EPOC, bronquiectasias), enfermedad cerebrovascular, diabetes mellitus, neoplasia, insuficiencia renal crónica, enfermedad hepática crónica, alcoholismo, malnutrición y estado postesplenectomia

- Antecedentes de hospitalización durante los últimos doce meses

- Frecuencia cardíaca $\geq 125$ latidos/min

- Presión arterial sistólica $<90 \mathrm{mmHg}$ o presión arterial diastólica $\leq 60 \mathrm{mmHg}$

- Frecuencia respiratoria $\geq 30$ respiraciones/min

- Temperatura $<37^{\circ} \mathrm{C}$ ó $\geq 40^{\circ} \mathrm{C}$

- Estado mental alterado o confusión mental

- Hipoxemia: $\mathrm{PaO}_{2}<60 \mathrm{mmHg}$ respirando aire ambiente

- Hipercapnia: $\mathrm{PaCO}_{2}>50 \mathrm{mmHg}$ respirando aire ambiente

- Sospecha de aspiración

- Leucocitosis $>30.000$ elementos $/ \mathrm{mm}^{3}$ o leucopenia $<4.000$ elementos $/ \mathrm{mm}^{3}$

- Función renal anormal: nitrógeno ureico $>20 \mathrm{mg} / \mathrm{dl}$ o creatininemia $>1,2 \mathrm{mg} / \mathrm{dl}$

- Anemia: hematocrito $<30 \%$ o hemoglobina $<9 \mathrm{~g} / \mathrm{dl}$

- Radiografía de tórax: compromiso radiográfico multilobar o bilateral, cavitación, derrame pleural, o rápida progresión radiológica

- Neumonía bacteriémica con hemocultivos positivos

- Neumonía por bacilos gramnegativos entéricos, K. pneumoniae y P. aeruginosa

- Admisión a la Unidad de Cuidados Intensivos

- Sitio de infección extrapulmonar

- Necesidad de ventilación mecánica

- Signos de sepsis o disfunción orgánica evidenciada por acidosis metabólica o coagulopatía

- Factores sociales desfavorables: ruralidad extrema, educación incompleta, falta de adherencia al tratamiento, trastornos psiquiátricos

- Imposibilidad de recibir tratamiento oral

pleural), exámenes de laboratorio (hipoxemia, hipercapnia, nitrógeno ureico elevado, anemia, leucopenia, leucocitosis, glicemia elevada) y microbiológicos (hemocultivos positivos, infección pulmonar por bacilos gramnegativos entéricos o $S$. aureus) [Ib, II, III].

Edad: Varios estudios han demostrado la asociación entre la edad avanzada y el riesgo de muerte en el hospital ${ }^{1-4,6,7}$ [Ia, Ib, II, III]. La NAC del anciano suele ser oligosintomática o se manifiesta con síntomas inespecíficos (confusión mental, rechazo alimentario, arritmias, insuficiencia cardíaca congestiva), dificultando el proceso diagnóstico y retrasando el tratamiento específico, comprometiendo adversamente el pronóstico de los enfermos [Ib, II]. La ausencia de fiebre, el estado de postración, las comorbilidades múltiples, los trastornos nutricionales y la procedencia de centros geriátricos constituyen factores de mal pronóstico [IIb, II]. Sin embargo, basados en la evidencia de los estudios clínicos, no existen razones que sustenten el empleo de variables clínicas diferentes para evaluar la gravedad en la población senescente [C].

Comorbilidad: La presencia de cardiopatía coronaria [III], insuficiencia cardíaca congestiva [Ib], enfermedad cerebrovascular con secuela motora o deterioro psicoorgánico severo [IIb, III], diabetes mellitus [Ib, II], enfermedad respiratoria crónica (EPOC, bronquiectasias) [II], cáncer [Ib], insuficiencia renal crónica [III], alcoholismo [III] y enfermedad hepática crónica [III] son factores predictores de mortalidad en neumonía del adulto ${ }^{1-4,7}$. Sin embargo, la contribución de las distintas comorbilidades a la gravedad de la NAC del adulto ha sido difícil de 
establecer, debido a la falta de uniformidad en la definición de las enfermedades crónicas en los distintos estudios y a problemas con la estratificación de la gravedad de las distintas comorbilidades. Esto podría explicar parcialmente el bajo poder predictor de las comorbilidades específicas como factor de riesgo de muerte en los análisis multivariados, a pesar del gran número de estudios que han demostrado su importancia en el análisis univariado.

Frecuencia respiratoria: Sin considerar la edad del enfermo, la presencia de taquipnea constituye uno de los indicadores más confiables de gravedad de la neumonía en los análisis univariados y multivariados ${ }^{4,7}$ [Ia, Ib, II, III]. Se ha descrito una relación lineal entre la frecuencia respiratoria y la mortalidad por neumonía. Sin embargo, en la práctica clínica, se recomienda que la frecuencia respiratoria mayor de 20 respiraciones/min sea considerada un signo de gravedad confiable en los pacientes con neumonía [C].

Estado mental: El estado mental alterado ha sido identificado como un factor de riesgo independiente de muerte en varios estudios, incluso en la población senescente ${ }^{4,7}$ [Ia, Ib, III]. Sin embargo, la definición del estado mental alterado ha dificultado el análisis e integración de los estudios pronósticos. A pesar de esto, el compromiso de conciencia cuantitativo y/o cualitativo constituye un excelente predictor pronóstico en pacientes con NAC [B].

Presión arterial: La hipotensión arterial sistólica (PAS < $90 \mathrm{mmHg}$ ) o diastólica (PAD $\leq$ $60 \mathrm{mmHg}$ ) y la presencia de shock séptico en la admisión al hospital constituyen factores independientes de mal pronóstico en el análisis multivariado de varios estudios [Ia, Ib, II, III]. En la UCI, la presencia de shock séptico o hipotensión arterial sistólica prolongada durante más de 12 horas que no mejora con adecuado reemplazo de volumen y/o requiere fármacos vasopresores es un signo adicional de mal pronóstico [II].

Oxigenación: La hipoxemia y la administración de oxígeno con una $\mathrm{FiO}_{2} \geq 0,5$ para mantener una adecuada oxigenación tisular o la aplicación de PEEP son indicadores de mal pronóstico [Ib, II]. La insuficiencia respiratoria aguda grave y la necesidad de ventilación mecánica en la admisión a la UCI o durante la evolución también son factores predictores de mortalidad [Ib, II]. La presencia de hipoxemia debe ser corregida de inmediato y es un factor determinante en la decisión de hospitalización de un enfermo particular.
Radiografía de tórax: Los infiltrados radiográficos bilaterales, el compromiso de más de dos lóbulos pulmonares, la presencia de cavitación y derrame pleural constituyen factores de mal pronóstico [Ib, II, III]. En el hospital y la UCI, la rápida progresión de los infiltrados radiográficos está asociado a mayor riesgo de complicaciones y muerte [II]. Sin embargo, la solicitud de radiografías seriadas para evaluar la evolución o gravedad del paciente hospitalizado no es recomendada fuera de la UCI, salvo que existan evidencias clínicas sugerentes de alguna complicación [C].

Leucocitos: La presencia de leucopenia (menos de 4.000 leucocitos $/ \mathrm{mm}^{3}$ ) o leucocitosis (mayor de 20.000 leucocitos $/ \mathrm{mm}^{3}$ ) en la admisión al hospital se han asociado a mortalidad elevada en el análisis univariado [III]. Sin embargo, el resultado de los análisis multivariados ha sido controversial y sugiere que la leucopenia podría ser un mejor predictor de mortalidad [Ia, Ib].

Función renal: El nitrógeno ureico elevado en la admisión al hospital ha sido descrito como un factor de mal pronóstico en pacientes con NAC en los análisis univariados y multivariados, probablemente reflejando el deterioro de la perfusión tisular ${ }^{1-4,7}$ [Ia, Ib, II, III]. Es importante destacar los principales exámenes de laboratorio que orientan al clínico acerca del riesgo de muerte o curso complicado del paciente con NAC, junto con la radiografía de tórax, éstos son la gasometría arterial y la medición de la uremia [C].

Microbiología: La neumonía bacteriémica con hemocultivos positivos tiene dos a tres veces

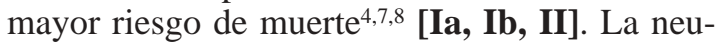
monía ocasionada por bacilos gramnegativos entéricos, Staphylococcus aureus y Pseudomonas aeruginosa suelen tener complicaciones durante la evolución y mayor letalidad [Ib, II]. La infección pulmonar por Legionella sp es causa frecuente de neumonía grave y admisión a la UCI en el extranjero, lo cual también ha sido comunicado en nuestro país ${ }^{9}$. Sin embargo, el cuadro clínico-radiográfico no ha permitido diferenciar entre los distintos agentes etiológicos de neumonía; de este modo, la información microbiológica tardía no ha sido útil para evaluar la gravedad del enfermo individual en la admisión al hospital o en el contexto de la atención primaria, ya sea en los consultorios o servicios de urgencia del país [C]. Los exámenes microbiológicos son útiles para evaluar la gravedad y orientar el tratamiento antimicrobiano en los pacientes hospitalizados por NAC [C]. 


\section{USO DE MODELOS PREDICTORES}

PARA EVALUAR LA GRAVEDAD DEL PACIENTE CON NEUMONÍA

La evaluación de la gravedad del enfermo con neumonía depende de la experiencia del médico clínico quien, se ha comunicado, suele subestimar la gravedad del paciente ${ }^{10}[\mathbf{I b}]$. Ningún factor pronóstico es suficientemente sensible y específico para predecir la evolución del paciente individual. De este modo, en la literatura médica se han descrito varios índices pronósticos que ayudarían al médico clínico a identificar a los pacientes con NAC que tendrían bajo o alto riesgo de complicaciones y/o muerte durante la evolución ${ }^{11-14}$. Ninguno de los modelos predictores desarrollados ha permitido estratificar a los pacientes en categorías de riesgo definidas [III]. La elaboración y difusión de guías clínicas que examinan la gravedad del paciente con neumonía mediante criterios objetivos, han permitido disminuir la hospitalización de pacientes de bajo riesgo, reduciendo significativamente el gasto de recursos médicos sin afectar la evolución y pronóstico de los enfermos ${ }^{15}$ [II]. Los modelos predictores basados en la gravedad son mejor visualizados como herramientas adyuvantes a la evaluación clínica de los enfermos. En general, los modelos predictores deben ser utilizados con prudencia y nunca deben predominar sobre el juicio clínico [D]. La evaluación de la gravedad periódica durante el curso de la estadía en el hospital es mandatoria para permitir el ajuste del tratamiento, evitando la morbilidad del tratamiento excesivo y/o las complicaciones del subtratamiento [D].

En la literatura médica contemporánea, se recomienda utilizar el Índice de Gravedad de la Neumonía descrito por Fine y cols para la pesquisa de pacientes de bajo riesgo, y el Índice de Gravedad de la Sociedad Británica de Tórax para la pesquisa de pacientes de alto riesgo con NAC grave $^{11-13}[\mathbf{C}]$. Basados en un estudio multicéntrico, Fine y cols han diseñado el Índice de Gravedad de la Neumonía (PSI) que permite estratificar a los pacientes en cinco categorías de riesgo de muerte ${ }^{13}$ [I Ib]. Los pacientes con neumonía en clase de riesgo I tienen bajo riesgo de muerte y eventos adversos, con una mortalidad que oscila entre 0,1 y $0,4 \%$. En un estudio observacional, los pacientes de bajo riesgo susceptibles de manejo ambulatorio presentaron una tasa de hospitalización dentro de los 30 días de $\sim 5,5 \%{ }^{16}$ [II]. Para identificar los pacientes de bajo riesgo es útil el modelo desarrollado por Fine y cols, quienes elaboraron un instrumento predictor en dos etapas para identificar a este grupo de enfermos. En una primera etapa, se consideran algunas variables epidemiológicas (edad, sexo, lugar de procedencia), la presencia de ciertas comorbilidades específicas (insuficiencia cardíaca congestiva, neoplasia, enfermedad hepática crónica, enfermedad cerebrovascular y enfermedad renal crónica) y algunos hallazgos en el examen físico (estado mental, frecuencia cardíaca, presión arterial, frecuencia respiratoria y temperatura). En general, los pacientes menores de 50 a 60 años, sin comorbilidad y/o anomalías en el estado mental y los signos vitales se clasificarían en las categorías de bajo riesgo, y se sugiere manejarlos en el medio ambulatorio, a menos que existan factores sociales que dificulten su control y/o adherencia al tratamiento (ej: alcoholismo, drogadicción, trastornos psiquiátricos, ruralidad extrema). Varios estudio internacionales que han validado esta clasificación sugieren que pueden identificar con bastante certeza tres categorías de bajo riesgo de muerte, a quienes se recomendaría manejar en el medio ambulatorio [Ib]. Estudios recientes han validado esta clasificación de la gravedad en adultos inmunocompetentes hospitalizados por NAC en el medio nacional ${ }^{14,17,18}$ [II, III].

Para identificar a los pacientes de elevado riesgo ha sido útil la regla discriminante desarrollada por la Sociedad Británica de Tórax (British Thoracic Society-BTS) ${ }^{11}$ que vuelve a confirmar que la frecuencia respiratoria superior a 30 respiraciones/min, presión diastólica inferior a $60 \mathrm{mmHg}$ y nitrógeno ureico superior a $20 \mathrm{mg} / \mathrm{dl}$ se asocian con mayor mortalidad [Ib]. La sensibilidad de esta regla es $88 \%$, la especificidad es $79 \%$, el valor predictor positivo es $19 \%$ y el valor predictor negativo es $99 \%$ [Ib, III]. Una modificación posterior de estos criterios ha incorporado la confusión mental como predictor y se ha comunicado que la presencia de dos o más factores de riesgo se asocia con un aumento de 36 veces del riesgo de muerte ${ }^{19}[\mathbf{I b}, \mathbf{I I}]$. La regla de la BTS modificada ha sido validada en diversos estudios extranjeros en población adulta y senescente ${ }^{20}$. Combinando los estudios de derivación y validación se han comunicado tasas de mortalidad ascendentes, correspondiendo a $2,4 \%$ en aquellos sin factores de riesgo, $8 \%$ cuando existe sólo un factor, $23 \%$ con dos factores, $33 \%$ con tres factores y $83 \%$ en pacientes con los cuatro factores de riesgo [Ib].

En el medio nacional se ha diseñado un instrumento pronóstico para evaluar la gravedad de 
los pacientes con NAC atendidos en los servicios de urgencia y consultorios de atención primaria $^{17}$ [III]. Esta herramienta incluye sólo variables clínico-radiográficas y no requiere exámenes de laboratorio complementarios como los índices pronósticos de Fine y la BTS. En el análisis multivariado, las siguientes variables clínicas resultaron factores predictores de muerte en el seguimiento a 30 días: presencia de comorbilidad, compromiso de conciencia, hipotensión arterial, taquipnea mayor de 20 respiraciones/ min y ausencia de fiebre.

El empleo del modelo predictor APACHE II en la evaluación de pacientes con neumonía grave en la UCI ha demostrado su utilidad como predictor de mortalidad [II]. Sin embargo, no ha demostrado ser aplicable en unidades de menor complejidad del hospital. La aplicación de este instrumento pronóstico fuera de la UCI es difícil, consume mucho tiempo y resulta poco práctico [D].

\section{RECOMENDACIONES PARA \\ LA EVALUACIÓN DE LA GRAVEDAD EN LOS PACIENTES CON NEUMONÍA ADQUIRIDA EN LA COMUNIDAD}

Se recomienda a los médicos clínicos implementar una estrategia simple y práctica para evaluar la gravedad y riesgo de complicaciones de los pacientes con NAC atendidos en el medio ambulatorio y hospitalario [D]. Se sugiere clasificar a los enfermos en tres categorías de riesgo:

- Pacientes con bajo riesgo (mortalidad inferior a 1-2\%) susceptibles de tratamiento ambulatorio u hospitalización abreviada [Ib, II].

- Pacientes con alto riesgo (mortalidad entre 20 y $30 \%$ ) que deben ser manejados en el hospital y probablemente en unidades especializadas (Unidad de Intermedio o UCI) con criterios de neumonía grave [IIb, II]

- Pacientes con riesgo intermedio, con comorbilidad o factores de riesgo independientes de muerte, pero que no es posible clasificar en una categoría precisa. El juicio clínico es esencial para decidir el lugar de manejo y tratamiento del paciente con NAC, especialmente aquellos ubicados en esta categoría de riesgo intermedia [D].

En general, los pacientes menores de 60 años, sin comorbilidad específica y alteraciones de los signos vitales y estado mental, se recomienda su manejo ambulatorio considerando su bajo riesgo de muerte y complicaciones [C]. Los pacientes senescentes (mayores de 65 años) y/o con dos factores de riesgo de la BTS, se recomienda manejarlos en el hospital con criterios de neumonía grave [C].

En el medio ambulatorio, donde no se dispone de exámenes complementarios, se recomienda evaluar la gravedad de los pacientes con NAC considerando las siguientes variables [D]:

- Edad mayor de 65 años [Ib, II, III].

- Comorbilidad: cardiopatía coronaria, insuficiencia cardíaca congestiva, enfermedad pulmonar crónica (EPOC, bronquiectasias), diabetes mellitus, enfermedad cerebrovascular con secuela motora, insuficiencia renal crónica, enfermedad hepática crónica, alcoholismo, neoplasia activa [II, III].

- Estado mental alterado: somnolencia, sopor, coma y confusión mental [Ib, III].

- Frecuencia cardíaca > 120 latidos/min [Ib, II].

- Hipotensión arterial (PA < 90/60 mmHg) [Ib, II, III].

- Frecuencia respiratoria $\geq 20$ respiraciones/ min [Ia, Ib, II, III].

- En la radiografía de tórax: compromiso radiográfico multilobar o bilateral, presencia de cavitación o derrame pleural [I Ib, II, III].

- Si dispone de oximetría de pulso: $\mathrm{SaO}_{2}$ menor de $90 \%$ respirando aire ambiente [Ib, II].

- Presencia de comorbilidad descompensada [IV].

En ausencia de factores de riesgo se recomienda manejo ambulatorio, en presencia de un factor de riesgo se recomienda manejo ambulatorio o en el hospital según la experiencia previa y el juicio clínico, en presencia de dos o más factores de riesgo se recomienda referir al hospital (Figura 1) [D].

En resumen, cuando el médico clínico debe decidir el lugar de manejo del enfermo (ambulatorio o admisión al hospital) es importante considerar las variables clínicas y sociales implicadas en cada caso particular. Se debe sobretodo evitar que pacientes de riesgo elevado sean tratados ambulatoriamente, pero también es importante minimizar el número de pacientes de bajo riesgo que son ingresados en el hospital innecesariamente [D]. Los diferentes estudios realizados han permitido elaborar un listado de factores de riesgo que condicionan la necesidad de ingreso hospitalario y ayudan al médico clínico en la estimación de la gravedad del paciente particular [D]. El juicio clínico y la experiencia 


\section{Edad mayor de 65 años.}

Presencia de comorbilidad.

Estado mental alterado.

Frecuencia cardíaca $>120$ latidos $/$ min.

Hipotensión arterial (PA $<$ 90/60 mmHg).

Frecuencia respiratoria $\geq 20 \mathrm{resp} / \mathrm{min}$.

Rx tórax: NAC multilobar, cavitación, derrame pleural.

$\mathrm{SaO}_{2}<90 \%$ con $\mathrm{FiO}_{2}$ ambiental.

Presencia de comorbilidad descompensada.

Factores sociales y problemas de adherencia al tratamiento.

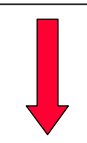

Ninguno

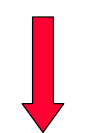

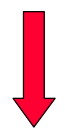

1 factor

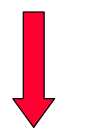

Juicio

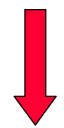

$\geq 2$ factores

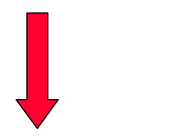

clínico
Manejo en el hospital
Figura 1. Recomendaciones para la evaluación de la gravedad del paciente con NAC atendido en el medio ambulatorio. del médico deben predominar sobre los modelos predictores, los cuales no son infalibles, y deberían siempre considerar las aspiraciones e inquietudes de los enfermos en la toma de decisiones acerca del lugar de manejo y tratamiento prescrito.

\section{BIBLIOGRAFÍA}

1.- BARTLETT J G, MUNDY L M. Community-acquired pneumonia. N Engl J Med 1995; 333: 1618-24.

2.- NIEDERMAN M S, MANDELL L A, ANZUETO A, BASS J B, BROUGHTON W A, CAMPBELL G D, et al. Guidelines for the management of adults with community-acquired pneumonia. Diagnosis, assessment of severity, antimicrobial therapy, and prevention. Am J Respir Crit Care Med 2001; 163: 1730-54.

3.- BRITISH THORACIC SOCIETY STANDARDS OF CARE COMMITTEE. British Thoracic Society guidelines for the management of community-acquired pneumonia in adults. Thorax 2001; 56 (suppl IV): 164.

4.- FINE M J, SMITH M A, CARSON C A, MUTHA S S, SANKEY S S, WEISSFELD L A, KAPOOR W N. Prognosis and outcomes of patients with communityacquired pneumonia. A meta-analysis. JAMA 1996; 275: $134-41$.

5.- GUEST J F, MORRIS A. Community-acquired pneumonia: the annual cost to the National Health
Service in the United Kingdom. Eur Respir J 1997; 10: $1530-4$

6.- FINE M J, HOUGH L J, MEDSGER A R, LI Y H, RICCI E M, SINGER D E, et al. The hospital admission decision for patients with community-acquired pneumonia. Results from the pneumonia Patient Outcomes Research Team cohort study. Arch Intern Med 1997; 157: 36-44.

7.- SALDÍAS F, O`BRIEN A, GEDERLINI A, FARÍAS G, DÍAZ A. Neumonía adquirida en la comunidad en el anciano inmunocompetente que requiere hospitalización. Cuadro clínico, factores pronósticos y tratamiento. Arch Bronconeumol 2003; 39: 333-40.

8.- DÍAZ A, CALVO M, O`BRIEN A, FARÍAS G, MARDONEZ J M, SALDÍAS F. Utilidad clínica de los hemocultivos en pacientes hospitalizados por neumonía adquirida en la comunidad. Rev Méd Chile 2002; 130: 993-1000.

9.- TRUCCO O, VICENCIO M, SALAMANCA L, OJEDA A, OYONARTE M, PRADO V. Participación de Legionella pneumophila en neumonía extrahospitalaria del adulto en Santiago. Rev Chil Infect 1993; 10: 89-95.

10.- NEILL A M, MARTIN I R, WEIR R, ANDERSON R, CHERESHSKY A, EPTON M J, et al. Community acquired pneumonia: aetiology and usefulness of severity criteria on admission. Thorax 1996; 51: 1010-6.

11.- HARRISON B D W, FARR B M, PUGH S, SELKON J B. Community-acquired pneumonia in adults in British hospitals in 1982-1983: a survey of aetiology, mortality, prognostic factors and outcome. Q J Med 1987; 62: $195-220$. 
12.- LIM W S, VAN DER EERDEN M M, LAING R, BOERSMA W G, KARALUS N, TOWN G I, et al. Defining community acquired pneumonia severity on presentation to hospital: an international derivation and validation study. Thorax 2003; 58: 377-82.

13.- FINE M J, AUBLE T E, YEALY D M, HANUSA B H, WEISSFELD L A, SINGER D E, et al. A prediction rule to identify low-risk patients with communityacquired pneumonia. N Engl J Med 1997; 336: 24350

14.- SALDÍAS F, MARDONEZ J M, MARCHESSE M, VIVIANI P, FARÍAS G, DÍAZ A. Neumonía adquirida en la comunidad en el adulto hospitalizado. Cuadro clínico y factores pronósticos. Rev Méd Chile 2002; 130: $1373-82$.

15.- ATLAS $S$ J, BENZER T I, BOROWSKY L H, CHANG Y, BURNHAM D C, METLAY J P, et al. Safely increasing the proportion of patients with community-acquired pneumonia treated as outpatients: an interventional trial. Arch Intern Med 1998; 158: 1350-6.

16.- MINOGUE M F, COLEY C M, FINE M J, MARRIE T J, KAPOOR W N, SINGER D E. Patients hospitalized after initial outpatient treatment for community acquired pneumonia. Ann Emerg Med 1998; 31: 37680.

17.- SALDÍAS F, FARÍAS G, VILLARROEL L, VALDIVIA G, MARDONEZ J M, DÍAZ A. Diseño de un índice pronóstico clínico para el manejo de la neumonía del adulto adquirida en la comunidad. Rev Méd Chile 2004; 132: $1037-46$.

18.- CERECEDA J, MATURANA R, ACEVEDO V, AYLWIN M, FLORES M. Índice de gravedad en neumonía comunitaria hospitalizada. Rev Chil Enf Respir 2003; 19: 155-9.

19.- KARALUS N C, CURSONS R T, LENG RA, MAHOOD C B, ROTHWELL R P, HANCOCK B, et al. Community acquired pneumonia: aetiology and prognostic index evaluation. Thorax 1991; 46: 413 8.

20.- LIM W S, MACFARLANE J T, BOSWELL T C, HARRISON T G, ROSE D, LEINONEN M, et al. Study of community acquired pneumonia aetiology (SCAPA) in adults admitted to hospital: implications for management guidelines. Thorax 2001; 56: 296301. 